\title{
AUTOMATED COSTRUCTION BY CONTOUR CRAFTING
}

\author{
Piyush Sharma ${ }^{1 *}$ \\ *1Department of Civil Engineering, Dronacharya College of Engineering Maharishi Dayanand University, \\ Haryana, India Email: piyushsharma1015@gmail.com
}

*Corresponding Author: -

Email: piyushsharma1015@gmail.com

\begin{abstract}
: -
Contour crafting $(\mathrm{CC})$ is a advanced building printing technology being researched by Behrokh Khoshnevis of the University of Southern California's in the Viterbi School of Engineering that uses a computer-controlled crane to build homes rapidly and efficiently with substantially less manual labor. It was originally designed as a method to construct molds for industrial parts. Khoshnevis decided to adapt the technology for rapid home construction as a way to rebuild homes after natural disasters, like the devastating earthquakes. Using a quicksetting, concrete-like material, contour crafting forms the walls of houses layer by layer until floors and ceilings are set in place by the crane. This great concept calls for the insertion of structural components, air conditioning, plumbing, wiring, utilities, and even consumer devices like audiovisual systems as the layers are built. Automation has been very advanced technology in manufacturing sector but the growth of automation in construction sector has been quite slow. Conventional methods of manufacturing automation cannot construct the giant structures with all internal features. This is the reason behind the slow rate of growth in construction automation. Contour Crafting has a great potential in automated construction of whole structures as well as its sub-components. Using this process, a single house or a colony of houses, each with possibly different designs may be automatically constructed in a single run. This research paper also deals with the application of CC in building habitats on other planets. CC will most probably be one of the very few feasible approaches for building structures on other planets, such as Moon and Mars, which are being targeted for human colonization. Contour Crafting is an emerging technology that uses robotics to construct free form building structures. The research paper aimed at providing a systematic solution for improving the overall Contour Crafting system efficiency in custom-designed buildings. The paper also deals with some practical construction issues.
\end{abstract}

Keywords: contour crafting, layered manufacturing process, construction automation, house construction, lunar construction.

\section{(우 $($ (1)}




\section{INTRODUCTION}

\subsection{General}

By the beginning of the twentieth century automation has grown and prevailed in almost all production domains other than in infrastructure. Implementation of automation in the construction domain has been slow due to:-

a) Conventional design approaches that are not suitable for automation.

b) Unsuitability of the available fabrication technologies for large scale products.

c) Smaller ratio of production quantity of final products.

d) Limitations in the materials that could be used by an automated system.

e) Due to expensive automated equipments.

f) Managerial issues.

g) Lack of technical efficiency.

On the other hand, the following are the serious problems that the construction industry is facing today: Accident rate at construction site is very high.

a) Labor efficiency is quite low.

b) Work quality is low.

c) Control of the construction site is difficult, and skilled workforce is less.

In the last two centuries, automation of various products has evolved considerably but very few of them are successful. Still construction of whole structures remains largely as a manual practice. This is because the various conventional methods of manufacturing automation do not fit in the construction of large structures. Layered fabrication is a promising new approach generally known as Solid Free Form Fabrication (SFF). Although several methods of SFF have been developed in the last 2 two decades and successful applications of these methods have been reported in various industries including industrial tooling, medical, toy making, etc., most of the current "layered fabrication" methods cannot deliver the wide variety of materials applicable to construction industry. Currently Contour Crafting (CC) seems to be the only layer fabrication technology that is uniquely applicable to construction of large structures such as buildings.

\subsection{The contour crafting technology}

The process involves feeding data to a machine that sprays and smooth out walls and structural components using nozzles, arms, and other tools. Khoshnevis' team imagines using this technology for commercial construction, low-income and emergency housing, and possibly space colony construction. Custom-designed structures can be automatically constructed by Contour crafting by repeatedly layering down construction material. Contour Crafting (CC) is an excellent fabrication technology that uses computer control for superior surface-forming capability in order to create smooth and accurate planes out of extruded materials. Some of the important aspects of CC are superior construction speed, lower construction cost, flexibility of architectural design, safety and friendliness to the environment. Multiple experiments have been conducted over the last few years to improve the $\mathrm{CC}$ process to produce a variety of small and full scale objects.

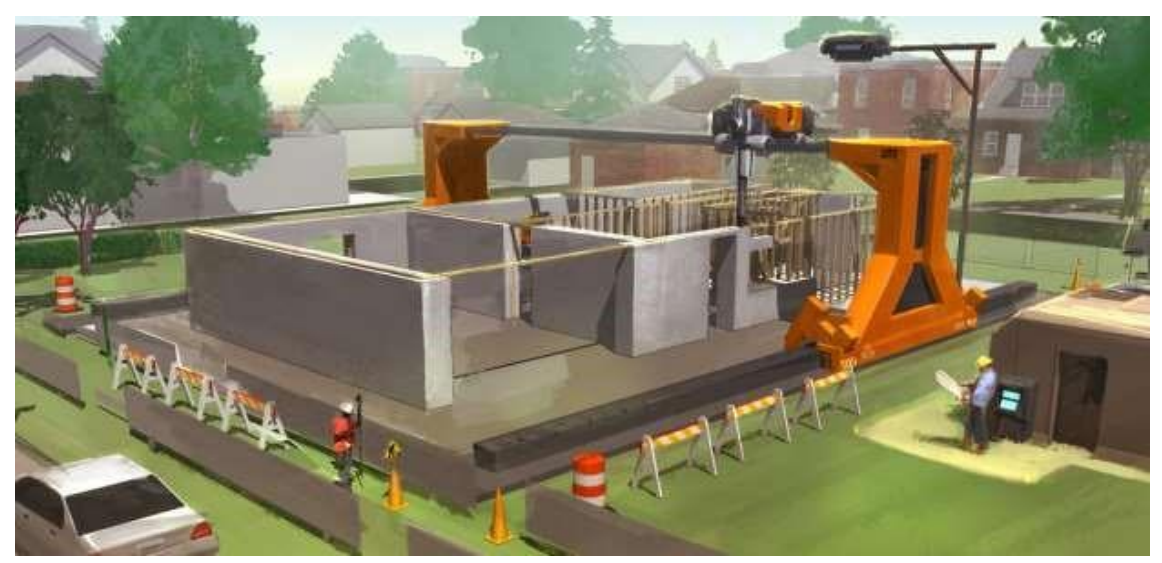

Figure 1: Contour crafting in construction.

The arms and nozzles used in the operation make multiple passes to reinforce the materials. Buildings are essentially assembled in layers automatically along a grid. One section of the machine moves vertically, while another moves horizontally. The speed at which a building can be built using this technology and the design quality of the finished product may enable it to become the construction industry's next big thing, according to Times Journal of Construction and Design. The benefits could include a safer work zone, speedy completion, and consistent quality. Machines do all the work with amazing efficiency, which could also mean significant cost savings through a decrease in human labor. Larger Contour Crafting have been developed to build larger structures. These machines combine a pouring process for forming the object surfaces and a filling process to build the object core. The $\mathrm{CC}$ machines are light weight and can be quickly assembled, disassembled and transported by a small crew. The construction operation can be fully automated requiring minimum supervision. 


\subsection{Planning And Optimization In Contour Crafting}

Tool path planning and optimization for Contour Crafting; benefit the technology by increasing the efficiency of construction of complicated structures. This research has intended to provide a systematic solution for improving the overall system efficiency and realizing the automation of the Contour Crafting technology for building the customdesigned houses. An approach is presented to find the optimal tool path for the single nozzle Contour Crafting system incorporating the physical constraints of the technology and construction considerations. Several algorithms have been given to find the collision-free tool path for the multiple nozzle system based on the single nozzle approach.

Operation planning and optimization play important roles in improving the overall CC system efficiency by generating optimal nozzle paths for the given structure designs. Multiple-nozzle or multiple-gantry systems is suitable for the construction of high-rise structures to reduce the construction time and cost. Two types of Multi-Machine CC systems are considered in this study:

Overhead multi-nozzle and multi-gantry, in both cases specific schedule and workload have to be assigned to individual nozzles or gantries for optimal operation. Collision between nozzles should be avoided without compromising the overall constructing efficiency.

\section{Contour Crafting}

The "Contour Crafting" (CC) is an emerging technology that produces a "print architecture".

This is done today via a kind of 3D printer (very large) and could be made tomorrow by robots of smaller sizes, the ground. In principle and with the suitable materials, the 3D printing can help to create entire buildings, as it is possible to create models. The same models can be made of sand melted by means of a lens focusing the sun's rays and computer controlled The first 3D printer by creating experiences of large architectural objects were conducted by Behrokh Khoshnevis of the Information Sciences Institute. He used it for a gantry crane and a computer-controlled to quickly create buildings in the future he would like to make inexpensive or requiring less material and less time, less labor with traditional construction methods. He also believes that this technology will build architectural objects on a massive scale. The key feature of CC is the use of two trowels, which in effect act as two solid planar surfaces, to create surfaces on the object being fabricated that are exceptionally smooth and accurate. Artists and craftsmen have effectively used simple tools such as trowels, blades, knives, and putty knives, with one or two planar surfaces for forming materials in paste form since ancient times. Their versatility and effectiveness for fabricating complex free-form as well as planar surfaces is evidenced by ancient ceramic containers and sculptures with complex surface geometries as well as detailed plaster work that have shapes as complicated as flowers, on the walls of rooms. Surface shaping knives are used today for industrial model making (e.g., for building clay models of car bodies). However, despite the progress in process mechanization with computer numerical control and robotics, the method of using these simple and powerful tools is still manual, and their use is limited to model building and plaster work in construction.

Generally automated systems allow an additive management, formative or subtractive matter. They can also combine in situ approaches. The CC method (Contour Crafting) suits the needs of a large construction tools software and hardware created to machine or produce molds for producing industrial parts for foundries, the glass or plastics and for 3D printers Since the mid-1990s, Khoshnevis gradually adapt these technologies to rapidly project to build a house, which could for example be implemented after natural disasters (tsunami, earthquakes) then even on other planets as part of the space exploration with human presence. In this, a fast-setting material such as concrete with sand and cement is used with a "setting accelerator", shape layer by layer in the walls and in some elements of home like floors, ceilings and roofing which are set up by the gantry or crane. Insertion of plumbing components, electrical and computer wiring, ventilation or insulation materials are provided upstream in the computerized plan, but the robot and its portico can also theoretically install pipes or some secondary structure elements or decorative elements and protection such as floorings, tiles, coatings, paints, etc.

The ability to fabricate extraterrestrial habitats, laboratories or manufacturing facilities is the key element for long-term human survival on the Moon or Mars. New proposal of NASA develops an automated in situ construction system that is viable, economical, practical within a decade for Earth-Moon operations.

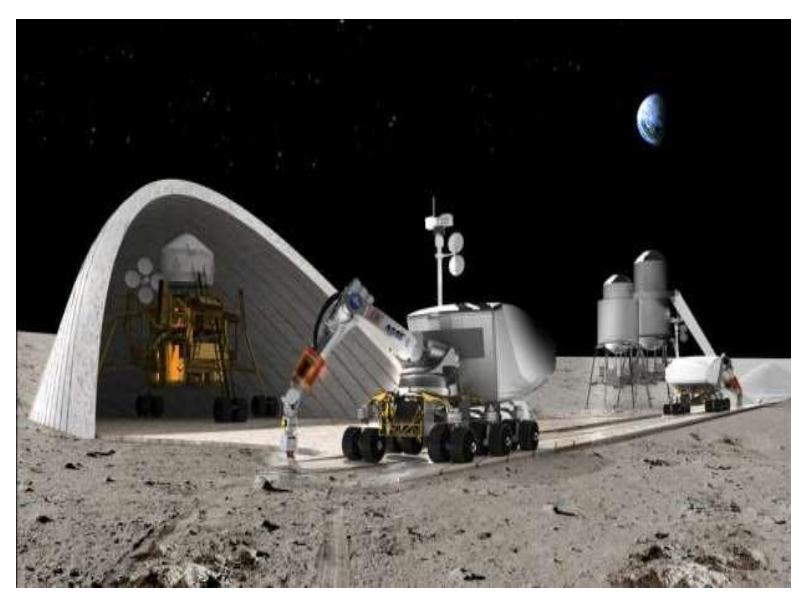

Figure 2: A picture view of contour crafting in space. 


\subsection{Objectives}

The objective of the work is to develop a systematic methodology for process planning and optimization for most efficient construction of complicated large-scale structures by Contour Crafting for single and multiple machines through the following steps:

1. Describe system characteristics and define tool path elements of Contour Crafting.

2. Develop practical tool path planning and an optimization method for the single nozzle CC system.

3. Develop practical tool path planning and optimization methods for multi-nozzle systems based on the optimization method for the single nozzle case.

\subsection{Some operational facts}

Operational facts are established according to the limitation and features of the system. The following are the operational facts for the system:

1. For every so many layers plumbing modules which have solder in the inner rim are assembled. Joints are made by electric heater which melts the solder

2. Conductive elements for power and communication lines are imbedded in polymeric blocks and are assembled robotically to form the desired electrical and communication networks.

3. Layers are similar to each other in 2.5D structures except at the points where openings for windows or doors are located.

4. The CC nozzle has to finish depositing one wall segment completely before starting a new wall segment.

5. In the construction process a nozzle has to completely finish a layer before moving on to the next layer. The only manual task is the installation of fixtures.

6. The nozzle will be free while traversing the places where windows or doors are located. This traversal time is called "air time"

7. Acceleration and deceleration times of the system are considered as fixed delays.

8. In order to avoid collision between the nozzle and the previously deposited walls by it, the nozzle should be lifted up when traveling between end points (at least one layer up).

9. The deposition flow rate can be perfectly controlled i.e., concrete flow can start and stop at any time.

10. In the multi-nozzle case the nozzles always work on the same layer. It is assumed that allowing the nozzles to work on different layers at the same time will decrease system efficiency.

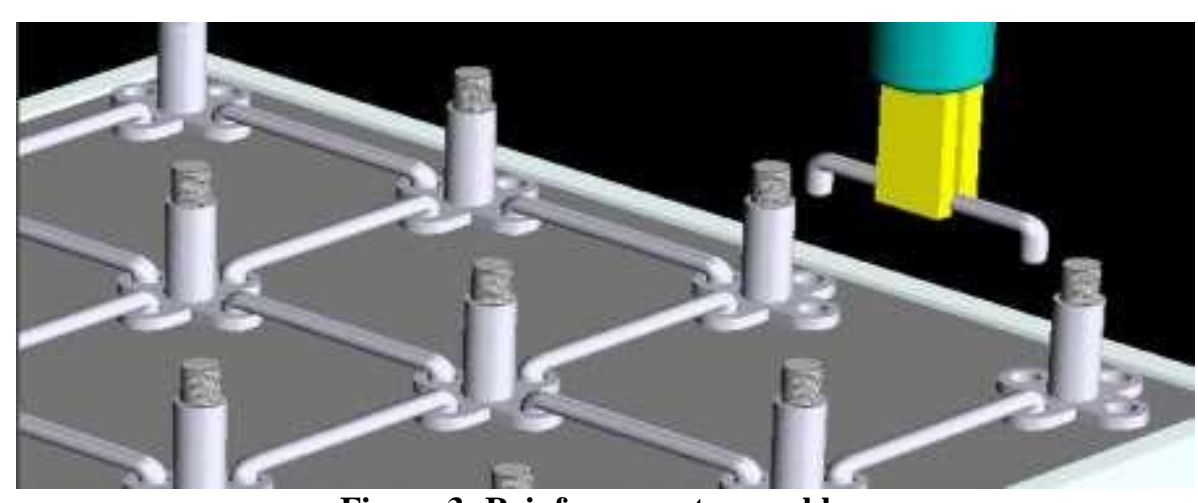

Figure 3: Reinforcement assembly

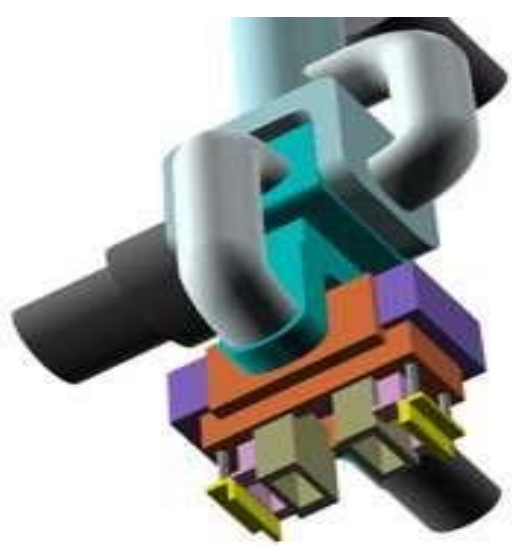

Figure 4: Slotted nozzle
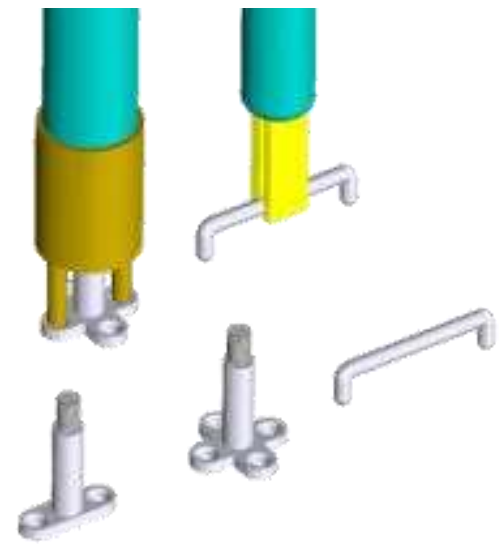

Figure 5: Assembly arms

\subsection{Logistics issues}

The new construction technology introduced by the Contour Crafting would necessitate a breakthrough approach to construction logistics. A technology that can build a house in one day requires a support logistics to procure and make available all the needed materials at the correct site in correct time sequence. A computerized system of bill of materials, 
order dispatching, transportation, and dynamic site layout planning will be needed. In addition, an infrastructure network with various warehouses with rapid loading capability must be implemented. The new technology will bring numerous logistics and optimization from high level planning to detail nozzle path panning and utilities module assembly planning research possibilities to the engineering profession.

\subsection{Trowel path planning and nozzle control}

The analysis deals about the ability to physically realize the task by specifying the trowel path, trowel/nozzle orientation, and nozzle flow control. At this level the constraints include collision avoidance of trowel/nozzle assembly with previously built portion of the structure, and minimization of layering imperfections (such as bulges, gaps and cracks). Again, we can potentially reduce these constraints to geometric reasoning problems by breaking each layering into smaller segments and then analyzing each segment. To extrude layers of more thickness, the volume of material extruded by the nozzle should be proportional to the speed at which the nozzle is displaced. Also, as the nozzle moves and extrudes, the trowel orientation must be tangent to the layering path at all times in order to form smooth surfaces. For straight or smoothly curved segments trowel orientation adjustments should be readily achievable.

Reasoning about corners: When the trowel reaches a corner it must change its orientation to be tangent to the opposite side of that corner. At such times the nozzle stops extruding as the trowel rotates and pushes the extruded material from the opposite edge. However, the push-in technique tends to create bulges around sharp acute corners because of excess material. The nozzle has to reduce its flow rate as it approaches the corner and the exact amount of material reduction can be computed based on the angle of the corner and geometry of the nozzle.

Process: The building model is first sliced into layers, and then the layout of one single layer is converted into a model which consists of edge and vertices. Edges represent the walls and vertices represent the intersections, corners or the end points of wall segments. A tool path of Contour Crafting for a particular structure must describe the position, orientation, velocity, and deposition rate of the nozzle in the entire construction period. Then this information is converted into a sequence of machine tasks and fed to the Contour Crafting machine. If time or energy spent on each machine task (such as deposition, nozzle traveling or nozzle rotation) can be considered as cost of construction, then process optimization will be; finding a path with the minimum total cost associated with every machine task. Therefore, costs of deposition, traveling and rotation need to be defined for calculating the overall cost associated with the tool path.

Cost estimation: Once the machine parameters have been defined, cost of deposition for each wall segment can be calculated according to its geometrical information. Cost of traveling between the edges is related to the cost of moving between the vertices and the cost of rotation along the edges. This cost can be estimated according to the relevant position of edges. The total construction can be evaluated once a tool path has been defined. Each edge has two end points; therefore, there are a total of four possible traveling costs from one edge to another edge. Since the nozzle of the Contour Crafting machine has to orient itself to be perpendicular to the tangent of the wall segment, the nozzle may need to be reoriented when traveling between edges. For example, in order to construct a corner, the nozzle must rotate $90^{\circ}$ between the constructions of two wall segments.

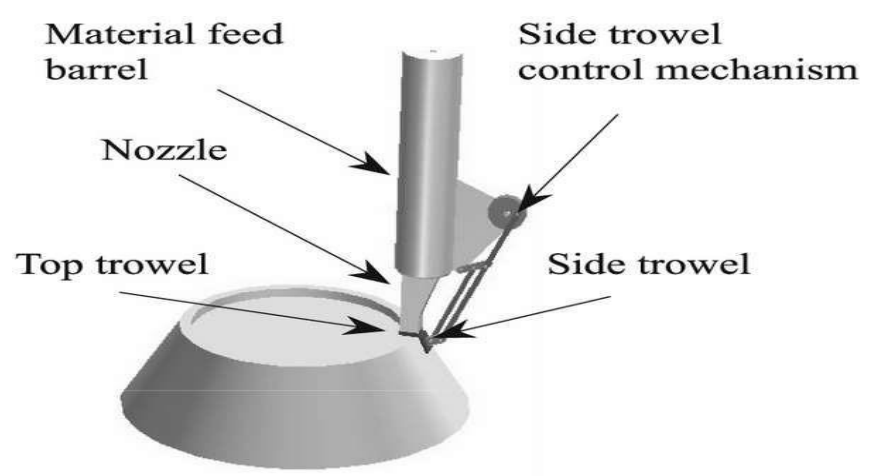

Figure 6: Tools used in contour crafting.

\subsection{Environmental aspect of contour crafting}

Every year in the developed countries like United States, 400,000 workers are seriously injured or killed doing construction work. Construction work is dangerous and involves too much risk for human life and human resources. Resulting litigation from work place injuries are increasingly raising the overall costs of construction. Waste is also a major concern for conventional construction methods. Construction of a typical single family home generates a waste stream of 3 to 7 tons. Globally more than 40 percent of all raw materials are consumed in the construction process. Construction, in addition to wasting valuable resources, contributes significantly to environmentally harmful emissions. There is a necessity to reduce the negative environmental impacts of construction and to eliminate worker safety hazards. Contour Crafting is a robotic technology with very accurate computer control. Due to the nature of this technology, materials are extruded with perfect precision and near zero waste. Contour Crafting offers construction without waste, 
noise, dust or harmful emissions. As a result construction by Contour Crafting will be an environmentally friendly and sustainable process. And since Contour Crafting is an automated robotics technology, workers will not have to risk life to do backbreaking and repetitive tasks. The resulting reduction in labor injuries and the subsequent litigations will reduce both the human and financial costs of construction. Contour Crafting will prove to be much safer for the worker and for the environment with these benefits:

1) Less total energy use for all construction activities

2) Less transportation of material, equipment, and people.

3) Less material and energy waste during construction.

4) Less total material use.

\section{During the building process:}

1) Fewer injuries in construction and transportation-related activities.

2) Less worker skin contacts with hazardous substances.

3) Less noise exposure.

4) More efficient buildings therefore less energy use in HVAC.

5) Efficient internal space use for occupants.

6) Less exposure to airborne substances such as dust and chemicals.

7) Easier to establish procedures for hazard and failure analysis during construction.

8) Increased structural strength and durability due to less aging material.

9) Increased seismic safety due to improved construction design and material.

\section{Negative impact:}

The other big drawback to contour crafting is the impact involved in the use of concrete, and more specifically, the cement used in the making of concrete. Both concrete and cement are mineral mixtures, so must be mined. While these minerals aren't exactly scarce, mining itself has severe negative impacts on the environment, and the use of concrete as the base material for the mass printing of homes would vastly increase the demand for minerals. Furthermore, the manufacture of concrete is one of the largest sources of atmospheric $\mathrm{CO} 2$, a major greenhouse gas, producing over $5 \%$ of all anthropogenic emissions. So while contour crafting might be an enticing socio-economic solution to the housing problem, it isn't feasible from an environmental standpoint, at least with current industry practices.

\section{Sustainability potential of contour crafting:}

With growing concerns regarding what are considered environment friendly methods and materials any new method of construction must take environmental concerns under consideration. Simply saving on construction cost is not enough to justify the new use of system. Contour crafting must have the potential of being employed as a sustainable alternative to traditional methods of construction with regards to carbon emission, embodied energy, life cycle costs and the use of recycled materials. While an often sitespecific integrated approach is required in order to any building to be less hazardous to environment as compared to other approaches, the method of construction chosen has a great impact on sustainability of any project. While no construction method can be considered as inherently sustainable, there are practices considered more sustainable than others. In order to further understand the sustainability potential in contour crafting in application to single family housing it was reviewed using the USGBC LEED for homes point system published in 2008 as a guideline. LEED was selected as a baseline since the guidelines set by LEED have become the current accepted international standard for defining sustainable building practices. Contour crafting reduces the amount of time spent on site thereby reducing the negative environmental impact of prolonged construction. Concerns such as silt and runoff due to disturb topsoil are reduced notably through diminished exposure to weather before being permanently reset into the landscape. Also the fewer times workers are needed on site and fewer times they must travel to the site and more efficient the construction process becomes. The amount of waste generated on site also reduces as CC process only mixes the cement and polymer right before the application. However due to the nature of contour crafting and requirements necessary for the construction process to be effective, other areas of LEED for homes became affected through the use of CC.

\subsection{Applications of contour crafting Commercial applications}

Contour Crafting can significantly reduce the cost of commercial construction. Projections indicate costs will be around one fifth as much as conventional construction. Contour Crafting promises to eliminate waste of construction materials. Contour Crafting construction projects will be extremely accelerated; for example a 2000 square foot house can be constructed in less than 24 hours. This rapid construction time minimizes the financing costs of construction projects that typically take six months or longer to complete. While the costs of manual labor will be significantly reduced, physical power will be exchanged for brain power in the construction industry. For the first time women and the elderly will be able to take part in the construction industry. Construction could become a consumer market, wherein a house or other structure could be designed and built by the family that will occupy it. Reduced costs and automated building will make construction accessible to anyone. 


\section{1) Space colonies}

Contour Crafting technology has the potential to build safe, reliable, and affordable lunar and Martian structures, habitats, laboratories, and other facilities before the arrival of human beings. Contour Crafting construction systems are being developed that exploit in situ resources and can utilize lunar regolith as construction material. These structures can include integrated radiation shielding, plumbing, electrical, and sensor networks. Contour Crafting (CC) is the only fabrication technology capable of building objects with large layer heights while maintaining near-perfect surface quality. CC uses computer control to exploit the superior surface-forming capability of troweling to create smooth and accurate planar and free-form surfaces out of extruded materials. With its relatively large nozzle orifice, CC offers important advantages, including better surface quality, higher fabrication speed, and wider choice of materials/additives. The ability to fabricate extraterrestrial habitats, laboratories or manufacturing facilities is the key element for long-term human survival on the Moon or Mars. Our proposal develops an automated in situ construction system that is viable, economical, practical and with applicability within a decade for Earth-Moon operations.

\section{2) Low-income housing}

Considering the alarming nature of the shelter problem facing the world at the onset of the 21 st century, traditional methods of meeting the worlds housing demands will not be sufficient. New and innovative means of constructing shelter must be considered. Contour Crafting technology is the best way to address the housing problem of the 21st century. Using materials readily available at the build site, construction by Contour Crafting can build dignified houses or colony of houses with all the utilities for electrical and plumbing in less than 24 hours (per house). Due to the nature of this Solid Free-From fabrication technology, there will be minimal waste of construction material and almost non-existent labor costs and labor injuries and fatalities which happen frequently at construction sites (500,000 people get seriously injured or killed in construction annually in the US alone, a country in which stringent safety codes are regulated). The potential of Contour Crafting to quickly and efficiently alleviate housing concerns cannot be overlooked. Contour Crafting is the construction method of the future.

\section{3) Emergency housing}

Contour Crafting technology can deliver strong dignified houses to disaster victims very rapidly. Construction by Contour Crafting can build a 2,000 square foot house with all utilities for electrical and plumbing in less than 24 hours. Contour Crafting technology is adaptable and can use in situ construction material, thus eliminating the need to transport materials long distances, saving the time and costs associated with transportation. Since Contour Crafting is an automated process, labor needs are highly minimized allowing relief workers to allocate their time and effort to rebuilding local infrastructure such as water sanitation and distribution systems, roads, electrical and communication systems as well as irrigations systems. In this way Contour Crafting has the potential of providing disaster survivors not only with dignified shelter, but also with more resources to rebuild their lives and their communities.

\section{Conclusions}

Due to its speed and its ability to use in-situ materials, Contour Crafting has the potential for application in low-income housing and emergency shelter construction.

1) The speed of operation results in a good efficiency of construction logistics and management

2) Construction of luxury structures with exotic interior designs involving complex curves and other geometries, which are expensive to build using conventional method, is another vital domain of CC.

3) According to various statistics the construction industry accounts for a significant amount of various harmful emissions and also generates a huge amount of solid waste.

4) Construction of a typical two storey house generates a waste stream of about 3 to 7 tons. More than $40 \%$ of all raw materials used globally are consumed in the construction machines built for Contour Crafting may be fully electric, emission free and also generate a very little waste.

5) The CC method is capable of completing the construction of an entire house in a matter of few hours instead of several months as commonly practiced.

6) The environmental impact of contour crafting is also noteworthy.

\section{Acknowledgement}

I would like to thank Prof. Meeta Verma, Prof. Anil Kumar Chottu, Prof. Sakshi Gupta, Dr. D.K Singh, Prof. R.C Sharma and Prof. M.K Sinha for the valuable discussions on the contour crafting.

\section{References}

[1] Danijel Rebolj, Martin Fischer, Drew Endy, Thomas Moore, Andrej Šorgo, "Can we grow buildings? Concepts and requirements for automated nano- to meter-scale building”, Advanced Engineering Informatics, Science direct, Vol.25, pp 390-398

[2] Behrokh Khoshnevis Professor, Industrial \& Systems Engineering University of Southern California Los Angeles, CA 90089-0193, khoshnev@usc.edu," Automated construction by contour crafting-Related Robotics and Technologies", Science direct Automation in construction, Vol.13, pp 5-19.

[3] C. Balaguer, M. Abderrahim, S. Boudjabeur, P. Aromaa, K. Kahkonen, S. Slavenburg, D. Seward, T. Bock, R. Wing, \& B. Atkin, FutureHome: An Integrated Construction Automation Approach, IEEE Robotics \& Automation Magazine (2002) 55-66. 
[4] I. Vizotto, "Computational generation of free-form shells in architectural design and civil engineering", Automation in construction, Science direct, Vol.16, pp 224-231.

[5] R.A. Buswell, R.C. Soar, A.G.F. Gibb, A. Thorpe, "Freeform Construction: Mega-scale Rapid Manufacturing for construction", Automation in construction, Science direct, Vol.16, pp 224-231.

[6] B. Khoshnevis and G. Bekey, "Automated Construction using Contour Crafting - Applications on Earthand Beyond," Proceedings of the 19th International Symposium on Automation and Robotics in Construction, Gaithersburg, Maryland (2002) 489-494.

[7] B. Khoshnevis, R. Russell, H. Kwon, \& S. Bukkapatnam, Contour Crafting - A Layered Fabrication Technique, Special Issue of IEEE Robotics and Automation Magazine, 8:3 (2001-a) 33-42

[8] A. Warszawski, \& R. Navon, Implementation of robotics in buildings: current status and future prospects, Journal of Construction Engineering and Management, Vol. 124, No.1 (1998) 31-41.

[9] J. Lenssen \& C. Roodman, Worldwatch Paper 124: A Building Revolution - How Ecology and Health Concerns are Transforming Construction, Worldwatch Institute, Washington, D.C. (1995).

[10] G. Zak, M. Sela, C. Park \& B. Benhabib, Layered-Manufacturing of Fiber-Reinforced Composites, Journal of Manufacturing Science and Engineering, ASME, Vol.121 (1999) 448-455. 